Julián Marcelo:

\title{
Debilidades, Amenazas, Fuerzas y Oportunidades (DAFO) en las redes so- ciales
}

Strengths, Weaknesses, Opportunities and Threats (SWOT) in Social Networks

\begin{abstract}
:
As well as a first step in strategic planning within production realm, a SWOT analysis of a system-processproject in relationship with its environment is often carried out. Correspondingly, the different types of social networks can also be regarded as socio-technological environments used by social groups in the development of different projects; each of them with its own Strengths and Weaknesses in the use of such networks, which in turn entail generic and specific Opportunities and Threats to these groups. Furthermore, each social network, seen as a set of techniques and users, adopts forms of communication which enable/encourage the emergence, consolidation or disappearance of certain organizational models, for instance, with a different degree of horizontality, hierarchical, permeable or manipulated by the groups. These models are analyzed using organizational schemes, as particularly studied by Mintzberg who considers their design parameters and contingency factors. The paper deepens the analysis of deviations risks in the very complex systems-processes-projects which can be originated by the users of social networks - though with a high degree of uncertainty -, as well as their contribution feeding back the development of types and forms of the own social networks. Moreover, special attention is focused on synergies of different intensity in the acceleration of real changes within such social networks and their generated relationships, and particularly towards the likely creation of new systems of relations (in material and intellectual production, distribution, etc.) in all fields of economy, sociology, politics and culture.
\end{abstract}

\section{Agenda:}

Introducción. El cambio como objetivo y como proceso para alcanzarlo

La r-evolución evoluciona

Oportunidades de las redes sociales para los movimientos

Debilidades de los movimientos en el uso de las redes sociales

Amenazas de las redes sociales para los movimientos.

Riesgo, complejidad e incertidumbre en proyectos de 'cambio'

Comunicación, organización, decisión

Conclusión 1: el proyecto 'cambio' para una democracia real

Conclusión 2: proyecto 'cambio' para un socialismo del siglo XXI

Epílogo 


\section{Author:}

Prof. Emeritus Dr. Julián Marcelo:

- Business Management Dept. - Facultad de Informática, Polytechnical University of Valencia, Spain

- 面 + 34 - 625 - 756391, $ه$ jmarcelo123@gmail.com

- Relevant publications:

- Riesgo y seguridad de los sistemas informáticos. ISBN 9788497053303. Universidad Politécnica de Valencia, 2003. http://www.casadellibro.com/libro-riesgo-y-seguridad-de-los-sistemas-informaticos/9788497053303/906798 


\section{Introducción. El cambio como objetivo y como proceso para alcanzarlo}

Dentro de un debate amplísimo -como es el paso de la indignación al cambio en sus aspectos éticos, políticos y estéticos, contando con las Redes sociales-, esta contribución se reduce a los aspectos políticos del cambio derivables de lo que explicitan los usuarios-pensadores de dichas redes sociales pertenecientes al propio movimiento social español de 'indignados' del 15 de mayo [de 1991] (en adelante $\# 15 \mathrm{M})^{1}$, precisando en lo posible el alcance de ese uso y sus perspectivas razonables.

Para aclarar conceptos, la Real Academia entiende por 'cambio' la "acción y efecto de permutar (..) cuando se transita de un estado a otro" (entre otras acepciones), lo que permite introducir la teoría de los proyectos [1] que se definen como procesos de cambio dirigido con cierto 'riesgo' de incumplimiento (fig.1).

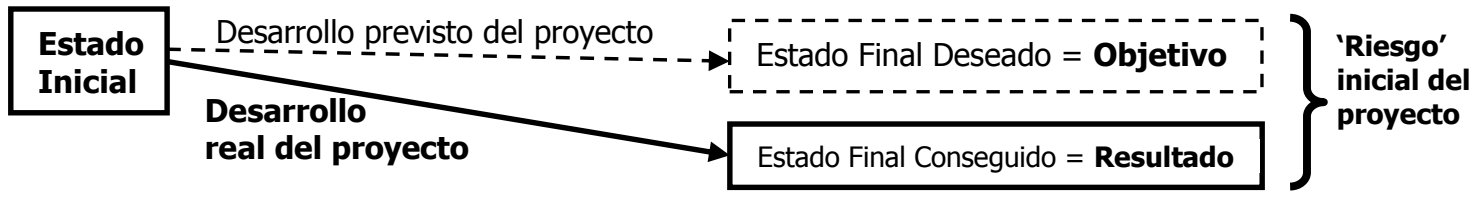

Figura 1. El proyecto visto como acción de cambio entre estados (Fuente: propia)

En cuanto un proyecto tiene alguna complejidad, su 'gestión' busca reducir ese 'riesgo' de 'no éxito' (o de incumplimiento) fraccionando el proyecto en hitos intermedios, cada uno con sus respectivos estados (deseado, conseguido, rectificado). En cada estado intermedio, dónde se alcanza ya un resultado desviado, el proyecto se rearranca empleando medidas potencialmente reductoras del riesgo, para conseguir, en el desarrollo subsiguiente del proyecto, un estado final resultante que se desvíe (del estado final deseado planificado inicialmente) menos que si no se hubieran aplicado dichas medidas [2] (figura 2).

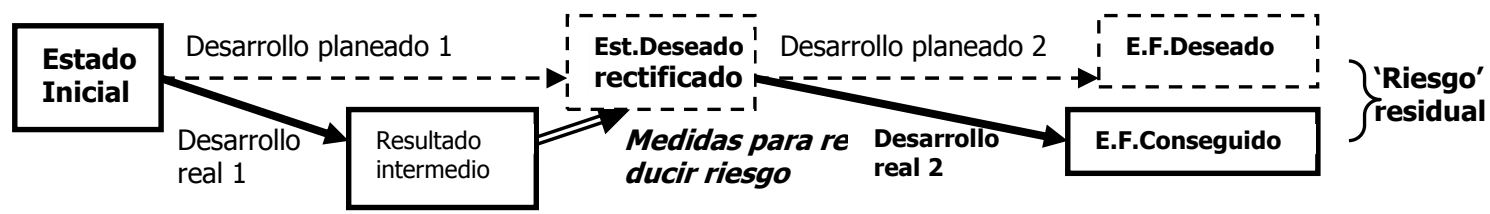

Figura 2. Gestión del proyecto reductora de su riesgo de incumplimiento (Fuente: propia)

\section{La r-evolución evoluciona}

El tránsito de la indignación al cambio, por parte del movimiento socio-político \#15M tomado como paradigma de indignación, es un macro-proceso-proyecto cuyo alcance debería poderse deducir de sus objetivos básicos a partir de las manifestaciones escritas de los 'indignados' en sus 18 meses de vida organizada que aquí se examinan. Escritos que van evolucionando, desde los producidos en los primeros meses de forma muy literaria por algunos de los autocitados como 'protagonistas' del \#15M (por ejemplo [3] o [4]); hasta los más recientes referidos al uso de las redes sociales como [5], dónde ya se conjuga la experiencia adquirida, junto a una mayor precisión instrumental. Se puede vivir así el paso de una primera etapa de movilizaciones indignadas y orientadas por objetivos iniciales, a otra que está fijando los suyos, con tensiones lógicas entre: por un lado, la fidelidad a los primeros (más o menos conseguidos y retocados); y por otro, las posibilidades futuras que el incuestionable 'éxito' de la movilización inicial permite entrever. Para analizar la evolución del proyecto \#15M, se parte aquí del primer 'manifiesto de los indignados' de la Puerta del Sol [4], materializado en 25 propuestas: 24 en 4 bloques y una final sobre feminismo igualitario.

\footnotetext{
1 Los informáticos del movimiento 15M lo denominan \#15M en su libro [5], que este artículo citará profusamente. Muchos de sus autores también usan el distintivo @ (@SuNotissima , @axebra, @quodlibetat, @democraciareal, @acampadasol, etc.) como una forma algo pedante, aunque hoy fácilmente comprensible, de autodenominarse en la era de la informática y así distinguirse como usuarios de las redes sociales, sea 'twiter' o la simple mensajería electrónica.
} 
- Un bloque de 6 propuestas sobre 'política real ya'se inicia con "la reforma de la ley electoral para que la democracia sea representativa y podamos participar". Y sigue con "poner fin a los privilegios de los políticos", "revocación de los mandatos", blindar los jueces "frente a las injerencias del poder político y del poder económico", "separación de poderes", "transparencia política para evitar el favoritismo y la corrupción"; o sea "reinventar el sistema político" pues "la sociedad está preparada para una nueva Asamblea Constituyente".

- El bloque central de 11 propuestas con 'SOL-uciones [!] económicas contra el malestar'exige que "las decisiones que no estaban incorporadas en programas electorales se sometan a consulta popular"; la "dación en pago y salir de la trampa de la vivienda en propiedad"; "luchar contra el fraude y la falta de corresponsabilidad de las grandes fortunas con la solución de la crisis"; "nacionalización de las cajas de ahorro y su reorientación" (hacia una "banca ética"); "reparto del trabajo, subsidio de paro, cumplimiento de los derechos laborales en su integridad, terminar con la precarización"; "comisiones de investigación de los organismos financieros"; "coordinación internacional para abolir los paraísos fiscales, imputación de las agencias de calificación, creación de una agencia pública de calificación"; "castigo impositivo a las empresas con comportamientos asociales y beneficios a las que cumplan con los principios de responsabilidad social", "democratizar las instancias financieras internacionales"; "tasa a los movimientos especulativos de capital"; y contra "el pacto del euro, los impuestos indirectos, el copago sanitario, la disminución de las prestaciones sociales, el reimpulso a las privatizaciones".

- Otro bloque de 3 propuestas sobre 'educación para indignarse' propone para la universidad "revisar el Plan Bolonia, aumentar becas y suprimir las becas-préstamo"; "educación pública, gratuita y laica"; y en los medios de comunicación, "contenidos que ayuden a entender la realidad y una mayor conciencia crítica, limites a la concentración, protección a la independencia del periodista y acabar con su precariedad".

- El bloque de 4 propuestas sobre 'los riesgos del mundo' se orienta a un "mandato: los países ricos tienen que reducir el consumo de muchos bienes"; así como a "prohibir los alimentos transgénicos y acabar el monopolio de las grandes superficies"; "consumir menos energía y menos perjudicial"; y a la "agroecología mediante el consumo local, ecológico y sostenible".

Este cuarto bloque de propuestas podría ser difícil de conseguir en el entorno del vigente sistema políticoeconómico capitalista global. Pero el resto de propuestas del manifiesto reproducido constituye un programa reformista, recuperador de cierto 'estado de bienestar' perdido por medio de una 'd-evolución' a la sociedad (más que revolución contra/en el sistema). Para conseguirlo, una mezcla de espontaneidad -tan deseada como teorizada- y de somera estructuración 'natural' se formaliza en "inventarse una nueva manera de discutir. Horizontal, asamblearia, con tiempo para consensuar las propuestas de mínimos y marcar horizontes de máximos. Comisiones para tratar las medidas inmediatas, y otras con menos prisa para llegar más lejos (..) Había que inventárselo todo y organizar el tráfico interno de la plaza, ocuparse de los más mínimos detalles del nuevo orden (..) En menos de 24 horas, en la Puerta del Sol se crea la estructura más compleja de comisiones y grupos de trabajo que se haya conocido en una movilización (..) Como si se fuera a abolir el dinero en aquel instante, o el trabajo obligatorio al día siguiente, como si quedaran apenas 7 días de plazo para arreglarlo todo, nadie más tenía 'nada más' que hacer que estar allí (..) El espíritu del recién bautizado 'mayo español' inauguraba una manera diferente de discutir (..) Es como una especie de terapia de grupo".

En estos primeros pasos del \#15M las líneas organizativas escasean, ya que al parecer sólo "se trataba de llegar a acuerdos con la responsabilidad de dotar de contenido las propuestas (..) No hay un centro, pero tampoco una periferia. Es una red que se tensa cuando se hace presión (..) En el magma inicial, todos caben. Pero el propio movimiento irá clarificando quién de verdad comparte el manifiesto de los indignados".

Un año más tarde, el folleto colectivo [5] empieza a explicitar la relación entre las formas de organización y de decisión ("llegar a acuerdos") con las formas de comunicación realizadas por medio de las redes sociales, como base de sus análisis "sobre la centralidad de las redes digitales en el \#15M"y en sus movimientos socio-políticos previos y consecuentes. Su introducción resume en cierta forma este primer tanteo de una marea creciente de encuentros-textos: "La sociedad deviene (en) red". Tras este impactante retruécano de la 'red social' como variante de la 'sociedad-red' de Castells, se enuncia cómo "en las últimas décadas, formas de organización nacidas EN internet [será CON o CON AYUDA DE Internet pues EN no es cierto ni 
técnica ni socio-políticamente] se multiplican y extienden por el territorio urbano, generando nuevas topologías, técnicas y políticas (..) Motores y síntomas de cambio en la historia moderna, las revoluciones y movimientos sociales pueden resultar ilustrativos a este respecto. La silenciada revolución islandesa, la celebrada primavera árabe y los movimientos como el \#15M y Occupy han confirmado la ascensión de nuevas formas de acción política en red. Se ha hablado de wikiRevoluciones, twitRevolution, Revolución 2.0... No siempre queda claro qué ideas, experiencias, tácticas o proyectos se esconden tras dichos eslóganes."

Para aclarar dichos proyectos o procesos y estudiar su adaptación a los objetivos propuestos, puede y suele emplearse instrumentalmente la técnica DAFO en todo tipo de espacios [6] y no sólo en los empresariales. DAFO parte de la situación o estado inicial del proyecto con un enfoque estratégico que tenga en cuenta las Fuerzas y Debilidades de la Entidad implicada (y que ésta puede manejar pese a su complejidad) con las que pueda aprovechar las Oportunidades ofrecidas por su Entorno y enfrentarse a las Amenazas de éste (menos manejables y por tanto más inciertas). La combinación de Oportunidades del entorno y Fuerzas propias constituye los Factores Críticos de Éxito FCE del proyecto en su dinámica. La entidad debe usar/potenciar esos FCE para enfrentarse a los Factores Críticos del Riesgo FCR del proyecto (no alcanzar los objetivos deseados), soslayando las Amenazas del entorno y reduciendo las Debilidades propias [7] (figura 3)

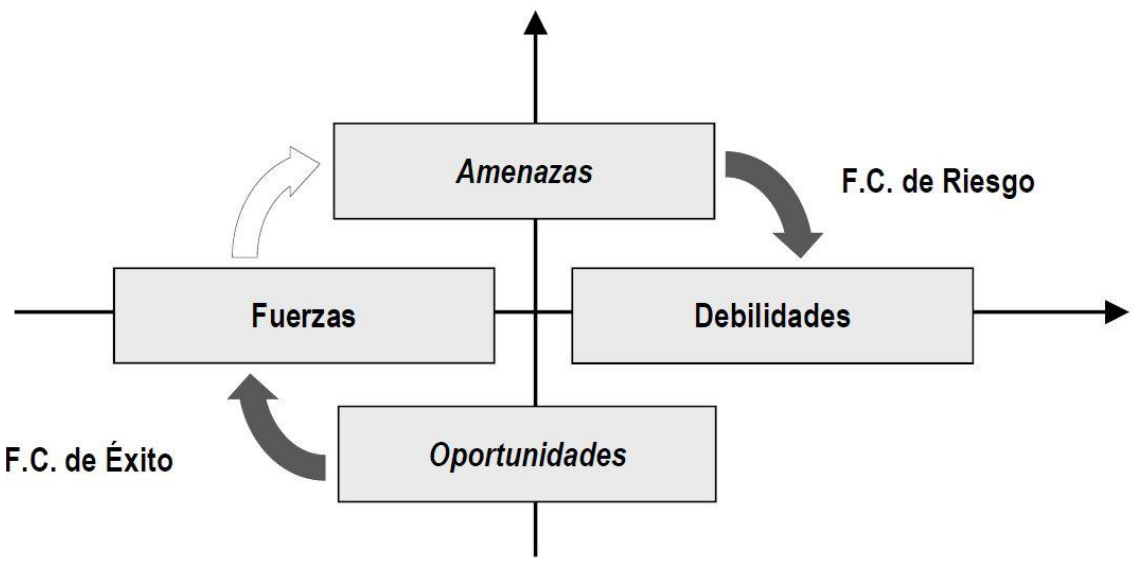

Figura 3. Esquema de confrontación dinámica de los Factores Críticos de Éxito y de Riesgo

Aquí se estudiarán, como entidades implicadas, los movimientos socio-políticos con sus Fuerzas (y sus Debilidades) para aprovechar las Oportunidades y enfrentarse a las Amenazas que les ofrecen las redes sociales que forman parte del entorno socio-tecnológico de dichos movimientos, centrándose en \#15M.

\section{Oportunidades de las redes sociales para los movimientos}

Todo movimiento sociopolítico está sostenido ineludiblemente por redes de comunicación que, al asumir su propia función dinámica, se convierten tautológicamente en redes sociales. Éstas han utilizado a lo largo de la historia 'lenguajes' -procedimientos de comunicación-, tan relevantes que se han tomado incluso como causa o consecuencia de los movimientos socio-políticos [8]. Desde el siglo XX ha ido creciendo la importancia de los 'lenguajes' de la Galaxia Von Neumann, hasta ocupar gran parte de la renacentista Galaxia Gutenberg y en agria competencia con la Galaxia Marconi [9]. Esta revolución tecnológica -por su generación- y socioeconómica -por su uso- procede de dos características básicas del lenguaje soportado informáticamente: su re-productibilidad a bajo coste, lo que dificulta su reducción a mercancía; y la re-simetrización del emisorreceptor, lo que no requiere la intermediación (con lo que este lenguaje se retrotrae en cierta forma a los de relación primitiva como el coloquial y mensajístico). Estas características sociales se completan con otras dos tecno-científicas que reducen el tiempo y amplían el espacio: la miniaturización portátil de los dispositivos de intercambio (basada en la nanomecánica química y la bidireccionalidad satélite-tierra); y la transmisiónmanejo-almacenaje de contenidos con algorítmicas revolucionarias de compactación reversible e indexación. 
No puede obviarse la capacidad del sistema capitalista para condicionar el desarrollo de esta nueva tecnocultura, sobre todo mientras se requieran concentraciones técnico-financieras en grandes servidores (por otro lado muy vulnerables y que podrán obviarse con técnicas de cloud-computing y uso cooperativo de capacidad de almacenamiento y transmisión excedente). Pero paso tras paso técnico, se constata que ha sido posible, en sólo dos décadas, una apropiación socio-técnica imparable de la red, con Internet como derecho humano básico, con software abierto, con comunidad creativa, con accesibilidad ética que alía privacidad personal y transparencia institucional. Esta conquista social ahora parece tan banal como para que quepa en un conectorcolector móvil -smartphone-tablet- de nueva generación, más o menos barato pero hiper-equipado con chips, gigas, banda ancha, pantalla táctil, cámara, android-iOS-x, GPS, apps... Una conquista que en todo caso requiere defenderla como cualquier otra frente a los intereses de sus enemigos ( $y$ así se ha hecho con notable éxito, impidiendo su mercantilización abusiva). Una conquista que, si probablemente ha pasado el punto de irreversibilidad, sólo anularía una hecatombe planetaria.

A partir del bagaje disponible de oportunidades que ofrece dicho entorno socio-técnico, cabe analizar sistemáticamente cómo el \#15M aprovecha este "conjunto de tecnologías y prácticas que apuntan a una reconstrucción de la acción y el espacio políticos" en la dirección que se deduce de los pronósticos hechos en 2011 por [4] y las afirmaciones hechas en 2012 por [5] "sobre la centralidad de redes digitales en el \#15M". Los indignados ya afirmaban en [4] la asociación del \#15M a la socio-tecnificación de la comunicación: "Si hace un siglo 10 personas podían reunirse para organizar un partido político, 100 años después hacen falta el mismo puñado de personas, pero el instrumento es una fluida comunicación en las redes sociales. Sobre esta base, DRY -Democracia Real Ya- tuvo la virtud de ir abriendo su propuesta sin perder la horizontalidad (..) Los enemigos de la Ley Sinde utilizaron la estructura de la red para armar una revuelta social que apenas ha costado mil euros. El apoyo de algunas organizaciones, grupos y redes de internautas permitió que la propuesta del 15M tuviera una gran repercusión (..) Quién nos hubiera dicho que esto que se articulaba en Internet sería la mayor movilización ciudadana que jamás haya tenido este país".

Jurado Gilabert (@SuNotissima) y otros destacan en [5] aún más a Internet, "no ya como sujeto central de una revolución tecnológica, sino como herramienta de transformación del marco cultural hegemónico cuando la red vehicula una profunda transformación, un auténtico cambio de paradigma, en los procesos comunicativos. Del esquema Emisor-Mensaje-Receptor, hemos pasado a un mapa complejo de multitud de emisores que, al mismo tiempo, se conforman como receptores, en la construcción conjunta y colaborativa de nuevos metarrelatos, que no tienen por qué coincidir (y no lo hacen) con la narrativa institucional que se viene reproduciendo desde las esferas de(I) poder y a través de sus medios de conformación de la realidad."

Que Internet y sus herramientas sean en su origen 'corporativas' (o sea propiedad de multinacionales y orientadas a los intereses del sistema) no importa en [5] para su uso progresista: "Que sean redes sociales corporativas no deja de resultar paradójico, si atendemos a los principios que guían al Movimiento (código abierto, Net Neutrality, conocimiento libre...). No sabría explicar este fenómeno sin otorgar al azar cierta dosis de protagonismo." @axebra lo ve dialécticamente: "la principal razón por la que han sido vertebradores de la acción colectiva es precisamente porque no estaban pensados para eso. Nadie visitaba las redes sociales esperando que arrancara una revolución. La gente las usa para expresar la complejidad de sus vidas: su trabajo, sus amores, su ocio, sus relaciones... eso genera relaciones de manera dispersa y amplia".

Facebook FB y Twiter TW bastaron para preparar el movimiento cuando "nacieron las comisiones y los grupos de trabajo (..) Se empezó a construir la infraestructura tecnológica de las acampadas, sus webs, sus espacios en FB y en la red $N$-1 (..) Los perfiles y canales de comunicación crecieron exponencialmente en cuestión de días y llegaron a tener cifras de seguidores realmente impresionantes. En FB, DRY tiene más 427.000 seguidores. En TW los perfiles más importantes del movimiento son el de @democraciareal con más de 118.000 seguidores, @acampadasol con más de67.000 y @acampadabcn con más de 40.000 (..) Estas redes sociales corporativas fueron reapropiadas para un uso político, a pesar de los problemas y riesgos que tienen. "Por su causa el \#15M buscó otros niveles tecnológicos, disponibles o a desarrollar. "Al mismo tiempo (..) las acampadas y DRY utilizaron herramientas creadas en base al software libre para organizarse: además de los blogs de 'tomalaplaza.net', ha sido 'N-1', una red social libre y autogestionada, que pasó de tener 3.000 usuarios antes del 15-M a más de 30.000 en apenas un mes (..) Se crearon herramientas digitales como 'propongo', 'stopdesahucios'y 'oiga.me' (..) que plantearon, tanto la autonomía de las redes digitales 
dentro del movimiento, como la capacidad para inventar dispositivos tecno-políticos que facilitarán las formas de decisión, organización y acción colectiva." El capítulo II de [5] detalla a 'Lorea', 'Takethesquare', '15octobernet' o 'Democracia4punto0', que aquí retomará el capítulo 6 sobre 'Amenazas'.

Fuerzas de los movimientos en el uso de las redes sociales

El \#15M presenta en [5] sus 'fuerzas' en el uso de las redes como ingentes e ilimitadas en el futuro. Sus portavoces tecnopolíticos han dado un enorme salto tecno-político que al menos ha superado décadas de desconfianza intelectual, filosófica y política [21] e incluso de crítica feroz izquierdizante contra la cibernética, informática, robótica y similares, vistas como negocios demoníacos manejados por reformistas vendidos al capitalismo, o al menos como denigraciones del papel central de las personas/masas en su liberación.

De forma tan excesiva como las condenas anteriores, Toret plantea en [5] que "quiero pensar la irrupción del \#15M dentro de una sociedad inmersa en un devenir-cyborg, como una transformación vertiginosa de los hábitos sociales y subjetivos debida a una profunda socialización en los mundos digitales interactivos, que genera nuevas capacidades que pueden expresar los cerebros y maquinas en red. La sociedad red vis/umbra nuevas formas organizativas y de contrapoder gracias al crecimiento de capacidades tecnopolíticas de las multitudes conectadas. Es la emergencia de nuevas formas de organización, inteligencia y acción colectiva. Comprender el 'cómo', las mentalidades, las capacidades y competencias colectivas del intelecto general aliadas a nuevas herramientas tecnológicas, puede crear nuevas formas de cambiar el mundo (..) El sistema-red $15-M$ se ha insertado dentro de una mutación de la subjetividad colectiva, de una politización del devenir cyborg de la sociedad, de una socialización en un ambiente cada vez más tecnológico que ataca a los intermediarios. Los cuerpos y cerebros siempre más inconfundibles [i!] con procesos de producción del trabajo vivo online, de información, de bits y de datos están profundamente conectados por sistemas de comunicación siempre más sofisticados. Por un lado, generan la nueva economía del capitalismo cognitivo, al mismo tiempo que pueden ser -como en la experiencia del \#15M- capacidad de autoorganización, innovación y autonomía de la cooperación común. Esa ambivalencia concierne a la potencia de transformación de las mentes conectadas en red, una transformación de los hábitos, de los usos de las herramientas digitales y canales de comunicación a partir del crecimiento, por momentos exponenciales de la auto-comunicación de masas o auto-organización común del intelecto general". Toret insiste en su visión ideológica al prologar su aportación con una cita de Franco Berardi 'Bifo', que ahora teoriza cómo "la crisis de la izquierda que se manifiesta en el retroceso político de las fuerzas organizadas del movimiento obrero $y$ progresista es solo el epifenómeno de un problema mucho más profundo: la crisis de la transmisión cultural en el pasaje de las generaciones alfabético-críticas a las generaciones pos-alfabéticas y configuracionales. La dificultad de la transmisión cultural no se reduce a una incapacidad de transmitir contenidos ideológicos o políticos, sino en la imposibilidad de poner en comunicación mentes que funcionan según formas diferentes y a menudo incompatibles." Según 'Rebelión' (16-08-2012) esta opinión excluyente es la que sostiene un sector político-ideológico citado como 'El autonomismo y su influencia en el 15-M'.

Sin llegar a este grado de converso, otros autores de [5] abarcan posiciones distintas, pero casi todas destacan incluso con soberbia las fuerzas con las que el \#15M usa las redes sociales, sin considerar más debilidades que las tópicas, como se verá. Pero además desligan el uso actual de las redes sociales de la persistente presión progresista mundial por el uso libre de Internet desde su creación. Ignoran también las movilizaciones por la informática y las libertades desde los años 80 en nuestro país; y minusvaloran en todo caso los antecedentes que no sean los directamente ligados a los grupos constituyentes del \#15M. Tanto @SuNotissima como @axebra recogen, como único antecedente a recordar, el "papel que ha tenido la lucha contra la 'ley Sinde' y por la neutralidad de la red como anticipadora del \#15M". Esta ha sido "la manera de aterrizar y cohesionar el descontento político, la indignación de millones de personas que se creían aisladas e incomprendidas, que ahora se han interconectado y puesto a trabajar, al unísono, por un nuevo mundo". Simona Levi es la única que recuerda como antes "se echa a un ministro en 2009 con la campaña 'Molina Pírate'." [el ministro que precedió a Sinde]. Porque @axebra ningunea las luchas previas a la ley Sinde "a la hora de desenmascarar las trampas de la llamada democracia o la ausencia de ella en nuestro país (..) Cuando los pequeños partidos se negaron en [sic] apoyar al Partido Socialista con su voto, los populares no tuvieron ningún problema en hacer un par de cambios estéticos a la ley y ayudar a su aprobación. "Reducir a lo inconcreto "los pequeños partidos" no sólo busca el 'apoliticismo' global más allá del bipartidismo turnante de gobierno. Lo quiere reforzar tecnopolíticamente, "porque Internet, por su propia condición no puede separarse en derechas o izquierdas, o de tal 
partido o tal otro, de tal manera que su transversalidad supuso una movilización también transversal". Toret incluso teoriza el poco peso que debe darse a los antecedentes: "La relación entre tecnologías y transformación social no es nueva, más bien lo contrario. Nos arriesgamos a plantear que sólo cuando los movimientos o agentes de transformación se anticiparon o superaron la apropiación por los 'poderes' de la comunicación y la tecnología se produjeron y aceleraron realmente los cambios sociales". Toret contradice así la visión marxista clásica en toda formación socio-económica de un desarrollo relativamente autónomo de las fuerzas productivas -en este caso las redes sociales y otros mecanismos relacionales-; desarrollo que entra en contradicción con las relaciones de producción y con todas sus superestructuras [14]. Esto no quita que el \#15M se apoye a menudo en los marxistas renovadores Deleuze y Guattari, citados incluso como fundamento de sus redes sociales abiertas y autónomas (RSAA) como $\mathbf{N - 1}$, "una de las redes de Lorea, proyecto que engloba varias redes sociales y busca su federación y también está enredado con Rhizomatik Labs. Es una noción utilizada por Deleuze y Guattari en el libro 'Mil Mesetas', en 'Introducción al Rizoma'o la multiplicidad no reducible al Uno. Es 'la resta que permite multiplicar'. Es el espacio de menos, que no suma dimensiones a un conjunto, sino que permite, a través del desarrollo de una interfaz herramienta compartida, componer $y$ recombinar en un común abierto." [5]

En esta línea de cierta inmadurez o dispersión ideológica totalmente comprensible aparecen contradicciones como las de @quodlibetat cuando busca superar las redes sociales corporativas RSC: "reivindicamos, en lo referente a la organización de la actividad de la inteligencia colectiva, el uso de RSAA, así como su federación, en base a principios colaborativos, participativos y horizontales, como sería el caso de $N-1$, Rise up, red DRY, o de herramientas como Diaspora o Identi.ca etc. Desde un punto de vista meramente técnico, las RSAA ofrecen una mayor diversidad de servicios de manera tal que se optimiza la organización de la actividad en red, así como su productividad en términos de eficacia y eficiencia respecto a las RSC. Desde el punto de vista ético-político, abogamos por las RSAA frente a las RSC, en tanto estas son privadas y privativas, es decir, en base al concepto de propiedad. Privadas entendiendo que no comparten su código, y por tanto limitan el acceso al conocimiento libre y compartido. Privativas dado que el acceso a ellas está condicionado a la aceptación de unas condiciones y términos de uso que, más allá de atentar contra la privacidad, suponen la cesión impuesta, la expropiación, de toda nuestra productividad inmaterial en la red, hasta el punto de no tener ningún derecho sobre esta, al mismo tiempo que se lucran de la gestión y explotación de nuestros datos y actividad sin que haya un retorno social o retribución".

¿EI \#15M no era anti-Ley Sinde? ¿O es que admite los derechos de autor, por supuesto de tipo copyleft?

\section{Debilidades de los movimientos en el uso de las redes sociales}

Los autores de [5] reconocen debilidades, que estudiarán mejor para reducir el riesgo de incumplimiento de sus objetivos. Para @axebra, "el riesgo de dispersión o de incapacidad de adaptarse al territorio es alto (..) También el riesgo de hiper-adaptarse hasta el punto de ser incapaces de proveer experiencias que puedan funcionar en otros territorios o llevados a cabo por otras personas (..) Si no es posible conectar esos nodos distribuidos de manera mallada (redundante) o p2p (comunicación organizada), el cultivo de experiencias puede caer en saco roto o desgastarse por la multiplicidad de convocatorias. El modelo copyleft se produce en otra escala, la del evento distribuido (..) Se es capaz de conectar, aunque sea momentáneamente, todas las luchas locales, estatales e internacionales en un corto espacio de tiempo donde se consigue dar una alta visibilidad. Esta acción, a pesar de su potencia visual, tiene el riesgo de caer en la propia de los eventos, que es su incapacidad para producir procesos (..) La potencia de este tipo de acciones vendrán marcadas por las fuerzas de los distintos nodos, pero sobre todo de la capacidad que tengan de 'romper la regularidad' (..) El objetivo de revolución global no debe extenderse a otros territorios sino extenderse a otras personas. La red es infinita en profundidad, de igual forma lo tiene que ser la movilización. No centrarse tanto en el número de ciudades sino ser capaces de inundar de conflicto los centros de trabajo, las escuelas y universidades, o las propias relaciones sociales. El dispositivo para llevarlo a cabo solo puede ser el que ha distinguido al propio 15-M, que es llenar un espacio de democracia radical y sobrepasarlo".

@SuNotissima también constata otra debilidad del movimiento: "El cómo la red se expresa en las plazas es, aun habiéndose avanzado mucho, una de las asignaturas pendientes del movimiento. Se pueden distinguir 
dos escalas bien diferenciadas dentro del 15-M. Una es el ámbito de actuación e interrelación puramente digital, mientras que la otra la conforman las personas que aún funcionan fuera de internet, únicamente en plazas, en la calle. Que existan aún muchas asambleas a las que les es ajena la manera de comunicarse en red ocasiona el que recurran a viejos esquemas organizativos, a saber, que las asambleas de una ciudad tengan que designar enlaces que asistan a coordinadoras para transmitir iniciativas e información; que éstas desciendan vía enlaces de nuevo a todas y cada una de las asambleas y que retornen a la emisora original, de nuevo, previo paso del dictamen por la coordinadora. Es fácil comprobar que estos procesos no se corresponden con la autodefinición de las asambleas como entes horizontales, pues la información traza claramente rutas de abajo hacia arriba y viceversa. De generalizarse aún más la comunicación vía internet, se podrían evitar estos caminos, donde sin duda se producen cuellos de botella, pérdidas de cantidad y calidad de la información y cierta posición de poder de aquéllos que ejercen de enlaces."

Simona Levi también analiza las debilidades del \#15M, pero desde el suelo, sincera, valiente y acertadamente. "En momentos de reflujo el espacio sano de la asamblea lo suelen ocupar 'reiteradores' que intentan convencerse a sí mismos que a fuerza de repetir las mismas palabras estas tiene vigencia, sin darse cuenta de que tan solo producen una fuga generalizada a otros espacios, dejándoles frente a una asamblea de simismos. Últimamente estamos diciendo: sé radical, pide lo posible. Aquíllega un aspecto confrontativo con un sector -pequeño, por suerte- del 15-M. Si se me permite una simplificación un poco caricaturesca debida a la exasperación por la cantidad de horas que hemos dedicado a debatir con él, este sector está constituido por los que consideramos el verdadero problema. Trolls aparte [los que publican mensajes provocativos o irrelevantes], estoy hablando por un lado de los radicales 'puros', que piensan que el sistema no se puede reformar -hasta aquí de acuerdo-, pero que también sabotean los intentos de utilizar demandas reformistas como arma de guerrilla; y, por otro lado, de los 'intelectuales críticos', que no se ven mucho por las asambleas. Nosotros pensamos que las demandas de reformas destruirán el sistema actual, porque el sistema está preparado para encarar enemigos pero no para que estallen sus propias contradicciones internas. Encarar el sistema a un imposible ontológico, 'destrúyete', ya le ofrece su defensa, la de ser tu antagonista. Si fuerzas a un sistema cerrado en torno a sus privilegios a 'mejorarse', solo le queda la deserción y la fuga como salida. Todo sabemos que al enemigo hay que dejarle una vía de salida si queremos ganar (..) No sabemos ganar. Quien magnifica el enfrentamiento no consigue 'hacer' porque el enfrentamiento es su 'hacer'. Cuando estamos ganando se deben abandonar posturas de trinchera (..) Lo que ha destruido grandes experimentos revolucionarios es el miedo interno a lo nuevo; por eso nos interesa la cooptación de nuestro mensaje y asumirla como una victoria. Quizás esto que voy a decir sea un poco 'islandés', pero lo comparto con mucha gente del 15-M. Si en tan solo un mes los políticos han dado ya varios pasos intentando copiar o aplicar nuestras demandas, nos debemos alegrar y marcarlo como victoria."

"(..) La acusación que más daño nos hace, ya que nos hace perder comunicación con gran parte de la gente, no es que seamos unos violentos, sino que seamos una chavalada que protesta sin nada que proponer $e$ incapaz de gobernarse (..) Los que niegan esta opción táctica luego defienden fervorosamente las protestas contra los recortes, como si esta no fuera la demanda más reformista y como si antes de los recortes el mundo hubiese sido bueno. Esta guerra es una guerra del lenguaje. El primer cambio ha de ser en el lenguaje, desde una profunda autocrítica. Ya no podemos complacernos en el martirio de pedir lo imposible; hemos de evolucionar de nuestro papel de antagonistas perdedores. Si el propio lenguaje que utilizamos es incomprensible es para hacernos los incomprendidos. Tenemos que ser responsables de nuestros actos. Si no somos leíbles para la mayoría, ayudamos a la fascistización de la sociedad. Por la euforia y luego la fuerza que nos dio vernos unidos en tan grande multitud en el movimiento 15-M, algunos están intentando imponer estéticas y lenguaje, dogmas que están en el tintero y que repiten como 'mantras' desde hace ya muchos años. Que se me entienda bien, por favor: una cosa son las palabras y otra las ideas, que pueden ser excelentes pero que a menudo se pueden expresar, digamos, con sinónimos, teniendo al fin y al cabo el mismo objetivo. El consenso masivo que hemos alcanzado con el movimiento 15-M justamente no viene de ninguna de las palabras que llevamos años repitiendo. Nos estamos confundiendo; no estamos ganando por lo que siempre hemos dicho, sino por lo que siempre hemos defendido. Primero viene la abolición práctica de privilegios y luego la justicia global. Así nació el 15-M y esta es su ola, queramos o no."

"(..) Hemos de estar presentes a todos los niveles. Debemos trabajar la implementación de la democracia directa pero también desmantelar el poder existente por dentro y su imagen mediática y memética. 
Hemos de ser tácticos con las palabras y con los actos. Pensar una acción por el resultado real y concreto que quiere obtener, no por razones viscerales o de justicia en abstracto... Si pides a la gente que odie su forma de vida se pondrán en tu contra; si compartes el odio por las mismas frustraciones, seremos invencibles. Como dicen los islandeses 'ocupar los medios para ganarse a la gente que mira la tele'. ¿No queremos este gran consenso? ¿Qué pasa?, ¿no queremos mezclarnos con el pueblo? Si usamos el lenguaje que entiende la mayoría, naturalmente estaremos usando un lenguaje copado por el sistema (..) La parte principal de juego es estudiar la ley, comprenderla, explicarla con otras palabras, ponerla en ridículo, hackearla para inutilizarla, destruir su autoridad sustituyéndola por otros cauces positivos que, finalmente, sean asimilados con el mal gusto y retraso que caracteriza al sistema."

\section{Amenazas de las redes sociales para los movimientos}

Los autores de [5] apuntan lógicamente que "debemos ser conscientes de las potencialidades y limitaciones de las herramientas y servicios de los que disponemos a fin de decidir los usos que le damos (..) La utilización de estas redes sociales corporativas fue relevante, aunque en nuestra opinión es importante problematizarlas, y a pesar de los problemas y riesgos que tienen, fueron reapropiadas para un uso político (..) Seguramente influyó el que gran parte de la población ya utilizase dichas redes, y desconociesen la existencia de otras herramientas más adecuadas, como las redes federativas de LOREA."Ésta se autodefine como "una comunidad de personas preocupadas por la seguridad, la privacidad y mantener el control de las herramientas de comunicación que utilizamos y los datos que compartimos en ellas. Por eso en las redes sociales de LOREA no hay usuarios, sino habitantes." Débil defensa si es sólo terminológica, frente a las amenazas a la seguridad y privacidad. Afortunadamente hay una preocupación real, "ya que sospechamos (y se empieza a ver) que, tanto FB como TW se verán presionados por los gobiernos para censurar comunicaciones que pongan en peligro su hegemonía. La indignación incluyente (Io que en occupy llaman «the 99\%») hace que estas redes se conviertan en el herramienta para expresarse y hacer crecer la indignación. Sin embargo, una vez estallada, estas dejan de ser útiles para organizarse. Las corporaciones que las poseen (en términos económicos) no van a apoyar esa revolución, y por lo tanto hacen falta otras redes para organizar la indignación como ha sido $\mathrm{N}-1$ (..) Sin duda, uno de los retos que se nos presentan es ir abandonando esas redes corporativas hacia otras emergentes como Diáspora o Identi.ca".

Esta sensatez contradice otras expresiones de autosuficiencia, como que "la práctica de la Tecnopolítica se da cuando se altera el uso habitual de Facebook y Twitter, para convertirlas en auténticas armas de difusión masiva de la información, o en útiles organizativos"(@SuNotissima). Es arriesgado olvidar que se aceptó el uso de Facebook por su difusión y facilidad de empleo, infravalorando su trayectoria sospechosa sobre todo si importa la seguridad y privacidad para la transformación de indignación en cambio. No sólo porque "las condiciones de la licencia ofrecen a FB la propiedad comercial de todo lo que tiene que ver con la vida privada de cada miembro de la red"ni porque sea casi imposible darse de baja. Porque Facebook (más aún que otras redes sociales corporativas) hace 'tecnopolítica', pero para los 'enemigos' del \#15M.

No puede olvidarse el análisis que hace Hodgkinson en 'With friends like these ...' ('Con amigos como éstos...) al indagar la propiedad de la CIA sobre Facebook:2 "Los vínculos de FB con la CIA pasan por Jim Breyer (uno de los tres socios clave que invirtió en esa red social 12,7 millones de dólares en abril de 2005), también asociado del fondo de capital Accel Partners, miembro de los directorios de gigantes como Wal-Mart y Marvel Entertainment y además ex presidente de National Venture Capital Association (NVCA), caracterizada por invertir en talento joven. 'La más reciente ronda de financiamiento de Facebook fue conducida por una compañía financiera llamada Greylock Venture Capital, que puso 27,5 millones de dólares.' Uno de los mayores socios de Greylock se llama Howard Cox, 'otro ex presidente del NVCA que también está en la junta directiva de In-Q-Tel', que 'es un fondo de capital de riesgo de la CIA,', creado en 1999 (2 años antes del 11-9) según su página web para "identificar y asociarse con compañías que estén desarrollando nuevas tecnologías para ayudar a proveer soluciones a la CIA". El origen de FB también se vincula con la renuncia del FBI a "su polémico programa de espionaje Carnivore creado para rastrear los correos electrónicos de internautas sospechosos en

2 The Guardian, 14-1-2008, http://www.guardian.co.uk/technology/2008/jan/14/facebook. Visitado el 25/11/2012. 
busca de palabras comprometedoras. The Register alude a dos documentos de 2002-2003 remitidos por el Centro de Información sobre la Intimidad Electrónica al Congreso de EEUU, dónde se detalla que el FBI no utilizó en dichos años 'Carnivore' ni su versión 'DCS-1000', recurriendo a programas comerciales de rastreo en las 13 investigaciones que llevó a cabo durante ese periodo". ${ }^{3}$ Además "el Ministerio de Defensa trabaja en un Carnivore europeo mejorado", aportando de 2007 a 2009 2.784.000 $€$, el 3\% de su Presupuesto. ${ }^{5}$

El análisis semántico de los contenidos que fluyen por las redes sociales, corporativas o no -para la discriminación por ideas y su posible neutralización y represión- es una amenaza con la que hay y habrá que contar cada vez más en el futuro. No es la única. La aplicación de 'matemáticas a la búsqueda del origen del rumor' lleva a la investigación de "un algoritmo capaz de localizar el foco las ideas que corren por Internet (..) El trabajo se basa en localizar a unos cuantos internautas centinelas, y aplica criterios como el tiempo de recepción o la cantidad de personas que están vinculadas a cada uno. Por ejemplo, para saber por qué todos los amigos de uno de repente empiezan a llamarnos Pepe -cuando siempre fuimos José- basta con fijarse en 15 de nuestros 500 mejores amigos (esos que tenemos dados de alta en FB). A partir de ahí, con tomar unos datos de sus cuentas se puede llegar al origen"; destacando como 'aviso a los navegantes' "su uso para llegar al núcleo de redes terroristas que se comunican mediante Internet o llamadas de móvil. Con tener a unos cuantos pinchados se podría llegar hasta el jefe. Cualquier sistema de divulgación en red es susceptible de ser descifrado con este algoritmo". 6 Por otra parte el jefe de los servicios secretos nos avisa: cuidado con lo que se habla por los móviles. En una jornada sobre 'Protección de la información sensible en Defensa y Seguridad', el general Félix Sanz Roldán avisa de que las informaciones que pasamos por teléfono pueden ser 'espiadas': 'Es sorprendente la ligereza con que utilizamos los teléfonos móviles, no en conversaciones, sino en el uso de sms".?

En definitiva, para saber si estos anuncios propagandísticos de invencibilidad son exageraciones o no (sobre todo en este periodo donde no se puede impedir ni el hackerismo) habría de llevarse a cabo el típico análisis 'cañón-coraza' sobre la hegemonía relativa entre lo ofensivo (amenazas) y lo defensivo (oportunidades) en materia de seguridad e intimidad de la red. En todo caso, todo movimiento-red que la utilice debe velar por el empleo de precauciones simples, recordables y en definitiva realizables en todos sus nodos [17].

\section{Riesgo, complejidad e incertidumbre en proyectos de 'cambio'}

Tras el somero análisis DAFO realizado y volviendo a la figura 3, ahora puede entenderse que las Debilidades de los movimientos sociopolíticos (\#15M en este caso) crecen con la complejidad de su sistema organizativo, ligado como cualquier otra organización a una dinámica de extensión geográfica, multiplicación de polos y profundización de actividades en grupos de trabajo (pese a la contención que en este caso implica el uso de las redes socio-políticas de comunicación). Este sistema organizativo ha de complicarse a su vez debido a sus actuaciones para controlar las Amenazas del entorno que constituyen dichas redes socio-políticas. Amenazas que producen cierta incertidumbre en cuanto a la selección y eficacia de las medidas que permitan gestionar -reducir- el riesgo del proyecto de 'cambio' del \#15M (en línea con las figuras 1 y 2 ).

La gestión del riesgo del proyecto de 'cambio' del movimiento se apoya en sus Factores Críticos de Éxito (sus propias Fuerzas y las Oportunidades del entorno de las redes socio-políticas) para reducir sus Factores Críticos de Riesgo (las correspondientes Debilidades y Amenazas). Pero por otra parte esa gestión debe tener en cuenta, como factores principales, la dinámica de la Complejidad organizativa del propio movimiento, así como la dinámica de la Incertidumbre ambiental que proceda de las redes sociopolíticas implicadas (además de contar

3 http://www.libertaddigital.com/internet/el-fbi-deja-de-utilizar-su-polemico-programa-de-espionaje-carnivore-1276241827/ Visitado el 25/11/2012.

4 El País, 22/02/2007.

5 www.la-moncloa.es/ConsejodeMinistros/Referencias/_2006/refc20061229.htm\#Inteligencia (citado por El País, 22/02/2007).

6 El País, 10/08/2012.

7 El País, 21/10/2009. El propio periódico cita como fuente: http://www.diariocritico.com/2009/Octubre/nacional/179028/cni-espionajemovil-sms.htm Visitado el 25/11/2012. 
con los recursos para realizar dicha gestión y de querer aplicarlos, por supuesto). [10] De forma más concreta: se ha realizado la exposición y análisis de los factores críticos (DAFO) que intervienen en el riesgo/éxito de cumplimiento del programa y de los objetivos del \#15M (o de cualquier otro movimiento socio-político) en la parte ligada a su empleo de las redes sociales que permean la sociedad-red.

Pero falta por estudiar el empleo de las técnicas de gestión de riesgos del proyecto que permita pasar de la indignación al 'cambio'. Esas técnicas requieren un control suficiente de la complejidad de la estructura organizativa que adopte el \#15M (de forma voluntaria o que se vaya desarrollando en él). Pero esto necesita cierto estudio de las estructuras organizacionales y de su dinámica (sea evolutiva o mutacional), como se indica en el capítulo 7 siguiente. Además dichas técnicas deben ofrecer la forma de enfrentarse dinámicamente a la incertidumbre generada en las redes sociopolíticas que van a utilizarse, lo que es mucho menos controlable, aunque factible, como se indicará en los capítulos finales 8 y 9 de conclusiones. [11]

\section{Comunicación, organización, decisión}

En [5] aparece a menudo "Organización... meta-organización... auto-organización... meta-comunicación... organización/comunicación (..) La decisión pasa por una interfaz que permite una permanente inclusión (autoagregación, auto-organización) y a la vez una regulación colectiva (no exenta de dificultades) de los pasos a dar (..) inventar dispositivos tecnopolíticos que facilitarán las formas de decisión, organización y acción colectiva (..) emergencia de nuevas formas de organización, inteligencia y acción colectiva." Los autores de [5] juegan frecuentemente con los términos de comunicación, organización y decisión, sin quedar claro cómo entienden y explotan las relaciones entre estas formas, que se vienen estudiando en la planificación estratégica de los proyectos-procesos desarrollada sobre todo para las empresas, lo que no impide utilizar con ventaja sus resultados en otros ámbitos como el de los movimientos sociopolíticos.

De entrada, la estructura de toda organización humana suele definirse como el conjunto de formas en que se divide el trabajo y se consigue su coordinación. El profesor Minztberg [12] parte de un sencillo esquema organizativo (véase el Anexo) basado en las relaciones entre grupos de personas agrupadas por sus intereses, afinidades y roles; bloques que pertenecen a dos grandes conjuntos: el dominio en estudio o sistema formado por grupos de intereses 'internos' a dicho dominio (directivos, apoyo técnico, apoyo administrativo, cuadros medios, operarios y sus mandos, así como cierta 'cultura' o ideología aglutinadora de la entidad); y el entorno de dicho dominio formado por otros grupos de intereses 'externos' (gobiernos, propietarios, clientes, proveedores, socios, competidores, sindicatos o asociaciones de empleados, público). Mintzberg desarrolla 6 tipos organizacionales básicos ligados al predominio que ejerzan los 6 grupos internos del dominio en estudio y añade como $7^{\circ}$ tipo la Organización Política, que aparece "cuando una organización no tiene una parte que predomine, ni un mecanismo de coordinación que sobresalga ni una forma estable de centralización. Probablemente tenga dificultad en ajustar los conflictos internos y su comportamiento se caracteriza por el impulso a la desunión". Otros autores añaden como $8^{\circ}$ el tipo de Organización Virtual [13].

En particular, la forma de toma de decisiones coordinable con la forma estructural de la organización está directamente ligada a su forma de coordinación-información-comunicación y ambas a su nivel de (des)centralización, vertical y/o horizontal. La búsqueda de descentralización en las formas de toma de decisiones con las redes sociales (que son los sistemas de información-comunicación de la 'organización' implícita en el movimiento \#15M) lleva a retener los tipos organizativos más descentralizados y más alejados de los mecanismos de coordinación por supervisión directa (la realizada por jefes o directivos segregados del conjunto organizativo).

El cuadro siguiente destaca 3 estructuras organizacionales de las 8 paradigmáticas de Mintzberg, que permiten combinarse y describir organizaciones reales como la asumida por el \#15M, como su dinámica (que suele desarrollarse hacia estructuras más complejas para enfrentarse a entornos más inciertos). El repaso de esas 3 estructuras será útil para prever la dinámica de los movimientos sociopolíticos: 


\begin{tabular}{|l|l|l|l|}
\hline Organización & Parte predominante & Mecanismo de coordinación & Característica básica \\
\hline Empresarial & Directivos & Supervisión directa & $\ldots$ \\
\hline Burocrática & Apoyo técnico & Normalización de procesos & $\ldots$ \\
\hline Divisional & Línea media & Normalización de resultados & $\ldots$ \\
\hline Profesional & Operarios y mandos & Normalización de habilidades & $\ldots$ \\
\hline Política & Todas por igual & - & $\ldots$ \\
\hline 'Misionera' & Ideología & Normalizacion de valores & responde a entorno hostil \\
\hline Innovadora & Apoyo + Operarios & Adaptación mutua & planificabilidad limitada \\
\hline Virtual & Sistema de información & Comunicación & red sinérgica +catalizadora \\
\hline
\end{tabular}

- En una Organización 'misionera', los miembros permanecen unidos en torno a los valores normalizados y tienden a una división del trabajo difusa y poco especializada. La socialización es clave para asegurarla.

- Una Organización Innovadora se estructura por proyectos y fusiona armoniosamente a expertos de diferentes especialidades en equipos creativos que usan la adaptación mutua y dispositivos de enlace.

- Una Organización Virtual potencia la comunicación de todos con todos y tiene los sistemas de información como centro neurálgico.

El complejo 'comunicación-organización-decisión' que explicita y al parecer experimentaría el movimiento \#15M, puede reflejarse como combinación de estos 3 tipos de estructuras organizacionales: 'misionera' (en torno a los valores ideológicos de su 'misión'), innovadora (coordinante de equipos creativos y de apoyos multidisciplinares que se adaptan mutuamente en sus discusiones y pueden replanifican constantemente sus proyectos) y virtual (apoyada en redes sociales de comunicación sinérgica y catalizadora de sus decisiones). Falta comprobar que esta tri-estructura deducible de las declaraciones tecnopolíticas de [5] puede conseguir una distancia-riesgo razonable entre los objetivos declarados del proyecto y el resultado futuro, centrándose en este caso en el papel que juegan y jugarán las redes sociales, como se verá en las dos conclusiones que siguen. ¿Cómo controlar su tendencia a la complejidad en el avance del proyecto? Pues podría resolver flexiblemente la mayoría de situaciones la continua preocupación por no salirse de las estructuras rízomáticas en el complejo de formas cohesionadas de comunicación-organización-decisión adoptadas.

\section{Conclusión 1: el proyecto 'cambio' para una democracia real}

Volviendo a la Introducción de [5], su final reinterpreta en clave técnica los objetivos sociopolíticos del \#15M: "La reapropiación multitudinaria de las redes sociales corporativas y la invención de nuevas herramientas libres, junto a estrategias hacktivistas a gran escala para fines de organización y comunicación político-vírica, han abierto un nuevo campo de experimentación socio-técnica. Este es el ámbito de la Tecnopolítica, como capacidad colectiva de apropiación de herramientas digitales para la acción colectiva, explorando esta nueva centralidad desde una visión tecnopolítica y tecnológica, pensando nuevas formas de organización/ comunicación así como la transformación subjetiva de los cuerpos conectados en red. Partiendo de una reapropiación de la política a la altura de los tiempos, este complejo movimiento ha mostrado la potencia de la cooperación en red como marco de apertura a nuevos horizontes políticos y colaborativos, desafiando los actuales sistemas de representación y apuntando a una posible r-evolución de la democracia."

La ' $r$ ' separable o conjuntable a evolución busca una ambigüedad calculada en los objetivos del movimiento y por lo tanto en el 'cambio' deseado. Puede interpretarse así razonablemente que el movimiento \#15M es una expresión organizada derivada de la indignación de las llamadas clases medias -sobre todo las juveniles- en su resistencia a la proletarización y su deseo de retorno a la situación previa a la crisis. Muchos interpretan así la composición y el discurso mayoritario de los grupos originales, acampados y portavoces del \#15M, así como su distanciamiento tanto de los partidos políticos gobernantes como sobre todo de los sindicatos mayoritarios con quienes no comparte historia, sin distinguir entre presuntos dirigentes institucionalizados y masas de afiliados reducidos a un status proletario que no desmiente un pasajero espejismo consumista. 
El \#15M estaría así dispuesto por ahora a un 'cambio' de tipo "sé radical, pide lo posible", como ilustran sus "dispositivos políticos para la acción colectiva" y siempre que se tuvieran en cuenta y se redujeran los factores críticos de riesgo considerados anteriormente (debilidades propias y amenazas desde el entorno digital). Este 'cambio' se centraría más en la participación social (modelizada en particular con 'Democracia 4.0' y 'Oiga-me') y en la vuelta a un 'estado de bienestar' recuperador del status de las clases medias y aceptable para el proletariado. O sea un sistema más avanzado que el de los años 90 pero sin los excesos 'burbujeantes' del primer decenio del siglo XXI, con las garantías que pueda dar un mayor control ciudadano y con los avances que pueden ir dando 'victorias' relativas como las consignadas por Simona Levi (siempre que las apoyen las movilizaciones específicas crecientes y los instrumentos como 'Stopdeshaucios'): "Quizás esto que voy a decir sea un poco 'islandés', pero lo comparto con mucha gente del movimiento del 15-M. Si en tan solo un mes los políticos han dado ya varios pasos intentando copiar o aplicar nuestras demandas, nos debemos alegrar y marcarlo como victoria. Con 'VdVivienda' no nos marcamos este tanto cuando la Chacón puso la ayuda para jóvenes"(pero que ha suprimido el PP al bajar la presión).

"La silenciada revolución islandesa (..) la transformación política de Islandia conocida como 'Revolución silenciada islandesa', que llevó a la cárcel los banqueros y políticos que llevaron a la quiebra al país". Estas y otras referencias al modelo islandés lo convierte para gran parte del \#15M en paradigma de revolución, frente a otras referencias más ambiguas o exóticas como la 'primavera árabe'. Pero no debe olvidarse cierta contestación descalificadora desde los 'media', con artículos como '¿Quién dijo que en Islandia hubo una revolución?'de Daniel López en [15]: "Me pregunto, pues, y lo hago sin retórica, quién se encarga de poner nombre a una revolución (..) He sido cuerpo indignado en la primavera española del 2011 y a día de hoy soy modesto aspirante a formar parte de la ejemplar comunidad islandesa, digamos que he trasladado mi indignación y mi hambre de una a otra de las zonas presuntamente revolucionadas. Pues bien, si he de ser honesto, debo decir que tanto aquí como allí no he conseguido aún vivir 'nihil novum sub solem' [nada nuevo bajo el sol] capitalista (..) que debo seguir siendo, aquí como allí, un perfecto adicto a la explotación, a ese veneno que matándonos nos permite vivir justo el tiempo que le somos necesarios."

\section{Conclusión 2: proyecto 'cambio' para un socialismo del siglo XXI}

Simona Levi, tan cauta e 'islandesa' (término que emplea ella misma), anuncia otra etapa al plantear "cuáles son las principales urgencias ahora, de cara a la \#globalrevolution": objetivo no explicitado del que sólo se intuyen rasgos a partir de problemas internos en el seno del \#15M: "1) Realizar un 'fork' [horquilla] entre 'imaginario islandés' e 'imaginario griego' para que ambos puedan convivir sin cortarse las alas. Lo que nos está resultando más difícil es luchar contra el proverbio 'mejor permanecer unidos'cuando la tensión interna es paralizante y cuando nuestra fuerza justamente es tener mil caras y mil nombres. "[5]

Otros autores de [5] también se preguntan "¿Qué formas de organización revolucionaria se van a dar en la sociedad red y el capitalismo financiero y cognitivo? ¿Cómo y de qué manera puede servirnos una politización del uso de las tecnologías de la comunicación para organizar el contrapoder en la sociedad red?". Preguntas que habría que precisar más ¿Solamente el "contrapoder"? ¿qué "mil caras y mil nombres"? ¿"tensión interna" entre quién? ¿Qué se está discutiendo? Al parecer, en el \#15M "hemos planteado algunas cuestiones como puntos de partida que abren los diálogos establecidos durante estos últimos meses. Las cuestiones permanecen abiertas como espacios de reflexión y a la vez nos orientan en el proceso de ensayo-error que permanentemente habitamos." Este clásico método para manejar la incertidumbre ofrecería cierta perspectiva de avance hacia esa 'global revolution' -con 'r'- que no se pare en una d-evolución a la islandesa, viendo a ésta incluso como etapa histórica necesaria para lo que viene a llamarse 'socialismo del siglo XXI' (una aproximación 'marxiana' a la complejidad, incertidumbre y crisis puede verse en [20]).

Los autores de [5] hacen sólo magras y fugaces referencias a la "socialización de una parte de la población (..) socialización en los mundos digitales interactivos (..) socialización en un ambiente cada vez más tecnológico que ataca a los intermediarios". Es lógico cuando los textos se centran en las herramientas tecnopolíticas que el \#15M y otros ya emplean y podrían desarrollar para ir superando sus debilidades reconocidas y desamenazando las redes socio-políticas. Pero empiezan a otorgar a dichas herramientas y redes una gran capacidad inexplorada de organización y sustentación del empoderamiento político, económico, social y cultural 
de los ciudadanos, de forma directa y no mediatizada, que permita generalizar una socialización cuyas características y viabilidad están en pleno debate.

Una etapa como la actual de desarrollo de la 'sociedad de la información' (o 'digital' para otros) permite sostener para muchos la visión marxista de una contradicción fundamental: entre el uso socializado de estas nuevas fuerzas productivas (y en particular el de las redes socio-políticas aquí analizadas) frente a la forma de propiedad de los medios de producción y distribución que sustenta la explotación del modo de producción capitalista y de los anteriores. No hay más que ver la persistente y no derrotada resistencia profesional y social que no permite la reductibilidad de la información, el conocimiento y sus derivados a ser una simple mercancía valorable más o menos por los mercados. Se abriría así (sin fecha) el camino político-económico a la 'sociedad del conocimiento' socialmente revolucionaria por su potencialidad reductora de una explotación que se apoya en las longevas superestructuras jurídicas, sociales y culturales de nuestro entorno.

\section{Epílogo}

El movimiento social \#15M parte de un proyecto de 'cambio' para una 'democracia real' y parece orientarse vagamente hacia alguna forma de 'socialismo del siglo XXI', según intuyen en [5] varios de sus portavoces tecnopolíticos. Pero ciertos extremos tecno-políticos como los que se ha visto que emplea Toret en anteriores páginas podrían implicar cierta deriva sociopolítica, en parte del movimiento, hacia una formación de tipo Partido Pirata (si el \#15M logra superar su prevención antipartidaria). ${ }^{8}$ Tras el PP sueco, ahora el PP alemán es, según [23], "un fenómeno fluido (..) Los Piratas nacen de las comunidades culturales de Internet, con personas de diferentes tendencias políticas. Les une la mutación del mundo del trabajo y el uso intensivo de Internet, que implica ciertos principios organizadores: transparencia, participación e igualdad. La campaña electoral de los Piratas apuntaba, en primer lugar, al modo de funcionamiento del sistema político y la forma de hacer política (..) La "protesta" del electorado de los Piratas se vuelve "contra el artificio ritual de una política cuya comunicación para muchos ciudadanos se reduce a los 'talk shows', a frases para la televisión o a comunicados que suenan a hueco (..) Los Piratas tienen tres áreas predilectas de desarrollo socio-político y las trabajan a la ofensiva: la utopía del libre acceso al conocimiento, la eficacia de los derechos de los ciudadanos y la implantación de prácticas democráticas." Para ellos, "la 'democratización de la democracia' significa ante todo que el problema no reside tanto en el sistema democrático como en su concepción actual. Su principal proyecto es desarrollar nuevas formas de participación política como respuesta a una crisis latente de la democracia representativa. Además de reivindicar más democracia directa y formas de participación no convencionales, añaden aportaciones de la tecnología como los open data o la open governance. Sin embargo, lo más importante, a sus ojos, es su propio trabajo sobre el proyecto de líquid democracy y líquid feedback (retroacción): un software experimental interactivo que permitirá recoger reivindicaciones y propuestas para facilitar el proceso participativo. La idea fundamental es la introducción del principio de la delegación del derecho de voto y la idea de "sufragio ponderado". Cada cual tendrá que decidir por sí y cada vez el nivel de su conocimiento sobre el tema y el grado de su participación (..) Los Piratas ponen en tela de juicio dos axiomas del sistema político actual: los partidos representan los intereses comunes de capas enteras de la población; y una organización debe integrar en un programa unitario la diversidad de los campos de acción política mediante la mayor eliminación posible de contradicciones (..) Creen que la diversidad de opiniones y el intercambio de puntos de vista diferentes por Internet es el mejor medio de elaborar estrategias políticas. Hay indicios para saber realmente que los Piratas han atraído a votantes descontentos, no porque fuera un nuevo partido, sino porque era un partido diferente."

Preocupa a los piratas "mantener la integridad de la información y poder determinarse libremente frente a la vigilancia del estado y la represión en una sociedad de comunicación digital (..) Detrás de la demanda de 'mantenimiento de los derechos burgueses a la libertad' se ocultan distintas posiciones: open source, open access, free culture, acceso libre al conocimiento son los conceptos esenciales para una nueva regulación de la Internet. En el centro están las condiciones de apropiación, las cuestiones de derechos de autor y los patrocinios. La posición de los Piratas no es homogénea. Hay quienes ponen el acento en la libertad de las

8 http://www.pp-international.net/ Visitado el 25/12/2012. 
condiciones de producción' y siguen comprometidos con la idea básica del copyright y la compensación financiera. La otra posición se refiere al principio del 'conocimiento libre' como patrimonio común de la humanidad, y rechaza la noción de 'propiedad intelectual', copyright o patentes de software propietario. Los productos de un trabajo de creación se consideran como 'creative commons".

"Esta idea de comunidad procede de dos principios fundamentales de la 'vida' de los Piratas: la idea de la cultura de Internet libre y la de unos 'bienes comunes' para todos en los campos de la cultura, del conocimiento, la genética, la biología y las infraestructuras públicas de agua, energía y derivados (..) La exigencia de esos 'bienes comunes' responde a la 'dialéctica de la precariedad' -en el trabajo, en la forma de vida- que se ha convertido en una situación normal. El mundo antiguo dónde el Estado garantizaba los derechos sociales de los trabajadores y la adquisición de bienes por el trabajo se ha convertido en un muy lejano, inaccesible. La falta de seguridad, la flexibilidad traen un alto grado de autonomía y libertad de elección, pero también obligan a encontrar continuamente un equilibrio frente a los excesos de trabajo, la irregularidad de ingresos y la disponibilidad exigida en cualquier momento. Si se quieren dominar los cambios venideros en el ámbito del trabajo y la vida personal sin que aumenten dramáticamente los gastos inducidos por estos cambios (prestaciones sociales, salud), hay que recurrir a ciertos 'bienes comunes'. Una renta mínima conviene más a identidades inseguras y heterogéneas que el establecimiento a largo plazo de las garantías que pueda dar un Estado social. No piden ya al Estado y a la sociedad más que estos 'bienes comunes'. En lo demás, cada cual se apaña. En Piratas, la imagen liberal-libertaria de los individuos y la sociedad participa a la vez del liberalismo y del socialismo, con una comunicación digital que en el futuro puede llegar fácilmente a áreas sociales diferentes (..) Una política de red en torno a la forma de derechos civiles y democracia penetra en las líneas de fractura social que la ciencia política sitúa en el enfrentamiento entre sistemas de valores autoritarios y libertarios. En realidad, estos polos están ocupados por los partidos tradicionales. Como éstos han perdido la oportunidad de entrar en la revolución digital o han dado a entender que este mundo cultural emergente les era bastante ajeno e indiferente, los Piratas pueden tener éxito y han encontrado su legitimidad política llenando ese vacío. Un vacío que podrán seguirlo cubriendo, ya que los partidos tradicionales no podrán llenar sus lagunas por una decisión brusca de cambiar de rumbo."

\section{References}

[1] Marcelo, J. Rodenes, M. Torralba, J. Estudio exploratorio sobre los métodos de gestión de Proyectos de alto riesgo. grupos.unican.es/igestion/socote/pdf/ActasCongreso1.pdf. Visitado el 25/11/2012.

[2] Marcelo, J. Sistemas, Proyectos y Negocios en la Sociedad de la Información. Jornada ATI-EOI, 2000. www.ati.es/gt/seguridad/PtoEncuentroAreas/JMCrsg_2000-11-07.pdf. Visitado el 25/11/2012.

[3] Alvarez K. y otros. Nosotros los indignados. Destino, julio 2011.

[4] Velasco, P. No nos representan. Planeta, julio 2011.

[5] Alcazan y otros. Tecnopolítica, internet y r-evoluciones (Sobre la centralidad de redes digitales en el \#15M). Icaria, junio 2012. http://www.icariaeditorial.com/pdf_libros/Tecnopolitica,\%20internet\%20y\%20r-evoluciones.pdf. Visitado el 25/11/2012.

[6] Portillo, C. Análisis DAFO: Uso de las Redes Sociales. 2011

[7] Marcelo, J. Dirección y Planificación estratégica de los Proyectos. Programa de doctorado del Departamento de Organización de Empresas. Master de Consultoría en ITIO (Integración de las T.I. en las Organizaciones). Universidad Politécnica de Valencia, 2006.

[8] Mcluhan, M. The Gutenberg Galaxy. Routledge \& Kegan Paul, 1962

[9] Marcelo, J. De la Galaxia Gutenberg a la Galaxia Von-Neumann. http://issuu.com/chasqui/docs/de-la-galaxia-gutenberg-a-la-galaxia-von-neumann. Visitado el 25/11/2012.

[10] ISO/IEC 31010. Técnicas de Gestión de Riesgos. http://www.previ.be/pdf/31010_FDIS.pdf Visitado el 25/11/2012.

[11] Fernández, M.; Marcelo, J. Driving IS projects, from research to practice. IFIP World Congress, 2008. http://dl.ifip.org/index.php/ifip/article/view/9881. Visitado el 25/11/2012.

[12] Mintzberg, H. El proceso estratégico, PHH, México, 1993. 
[13] Gil Estallo, Ma A. Dirigir y organizar en la sociedad de la información. Pirámide, 1999.

[14] Marcelo, J. Fuerzas productivas y relaciones de producción en la sociedad de la información. http://www.profesionalespcm.org/_php/MuestraArticulo2.php?id=10838. Visitado el 25/11/2012.

[15] Rebelión, 04/07/2012. http://www.rebelion.org/noticia.php?id=152474\&titular=\%BFqui\%E9n-dijo-queen-islandia-hubo-una-revoluci\%F3n?-. Visitado el 25/11/2012.

[16] Díaz Nafría, J. The need for an Informational Systems Approach to security. tripleC 9(1): 93-121, 2011. ISSN 1726-670X. http://www. triple-c.at. Visitado el 25/11/2012.

[17] Marcelo, J. MAGERIT y normalización de otros modelos de gestión de riesgos. Tecnimap 2004. http://www.yasni.es/ext.php?url=http\%3A\%2F\%2Fadministracionelectronica.gob.es\%2Frecursos\%2Fpae_000002318.pdf\&name=Juli\%C3\%A1n+Marcelo\&cat=filter\&showads=1. Visitado el 25/11/2012.

[18] Marcelo, J. Riesgo y seguridad de los sistemas informáticos. ISBN 9788497053303. Univer. Politécnica de Valencia, 2003. http://www.casadellibro.com/libro-riesgo-y-seguridad-de-los-sistemas-informaticos/9788497053303/906798. Visitado el 25/11/2012.

[19] Marcelo, J.; Teti, A.; Torralba, J. (Guest Editors). Advanced Information Systems Project Management. UPGRADE: Vol. VIII, issue no. 5, October 2007. www.cepis-upgrade.com http://www.ati.es/novatica/2007/189/Nv189-Presentacion.pdf . Visitado el 25/11/2012.

[20] Marcelo, J. Economía de la complejidad basada en la crisis vista como riesgo. http://www.nodo50.org/fimpv/ecompcri.pdf. Visitado el 25/11/2012.

[21] Matelart, A. Historia de la Sociedad de la Información. La Découverte, 2001; Paidós, 2007

[22] Marcelo, J. Nuevas Fuerzas Productivas frente a viejos modos de producción: proletarios y Sociedad de la Información. Publicado en 'Digitale Medien- Neue Möglichkeiten für Demokratie und Partizipation?' (Fleissner P. \& Romano V. Eds.) Berlin: trafo Verlag Dr. Weist 2007.

[23] Kahrs, H. Le succes du Parti pirate aux elections du parlement berlinois. Transform! 10, 2012 http://Www.espaces-marx.net/IMG/pdf/T_N10_Kahrs.pdf. Visitado el 25/11/2012. 